\title{
Criminologie
}

\section{Criminalité et répression de la criminalité au Japon : analyse socio-culturelle et criminologique}

\section{Hans-Heiner Kühne}

Volume 14, numéro 1, 1981

Criminalité et réalités sociales : Québec, Canada, États-Unis, Japon

URI : https://id.erudit.org/iderudit/017130ar

DOI : https://doi.org/10.7202/017130ar

Aller au sommaire du numéro

Éditeur(s)

Les Presses de l'Université de Montréal

ISSN

0316-0041 (imprimé)

1492-1367 (numérique)

Découvrir la revue

Citer cet article

Kühne, H.-H. (1981). Criminalité et répression de la criminalité au Japon : analyse socio-culturelle et criminologique. Criminologie, 14(1), 31-50.

https://doi.org/10.7202/017130ar d'utilisation que vous pouvez consulter en ligne. 


\section{CRIMINALITE ET \\ REPRESSION DE LA CRIMINALITÉ AU JAPON analyse socioculturelle et criminologique}

Hans-Heiner Kühne

Le constat d'une baisse de la criminalité, en particulier de la grande criminalité observée depuis plusieurs années au Japon a été a l'origine de nos travaux effectués en collaboration avec Koichi Miyazawa, de l'Universito Keio de Tokyo ${ }^{1}$. En effet, le Japon est aujourd'hui le pays industrialisé où le taux d'infractions est le moins élevé au monde. Dans une brève esquisse, nous presentons donc ici les résultats de nos recherches entreprises en 1977, lors d'un séjour d'études au Japon.

\section{LA CRIMINALItÉ JAPONAISE AU REgARD DES STATISTIQUES DE LA POLICB}

Toutes les données indiquées ci-dessous sont issues de sources en langue japonaise, en particulier du hanzai hakusho (le livre blanc sur la criminalité) et du hanzai toklisho (le rapport annuel de statistiques de la police). Les traductions anglaises des extraits de ces rapports ne correspondent pas toujours aux textes japonais, bien qu'elles ont été publiées officiellement par les ministères compétents. Ceci est également vrai pour les données communiquées à l'Interpol.

La qualification de l'infraction en droit pénal japonais est calquée sur le système juridique européen. Pour l'essentiel, les éléments constitutifs de l'infraction correspondent à ceux du code pénal allemand. Depuis la fin de la Seconde Guerre mondiale, la criminalité japonaise se développe selon un triple mouvement oscillatoire. En raison du désordre consécutif à l'aprèsguerre, la criminalité atteint son apogée en 1948 pour diminuer constamment jusqu'en 1953, puis progresser à nouveau de façon régulière jusque dans les années 70 . Le déclin progressif de la criminalité, constaté à partir de 1970 , sera néanmoins interrompu par une légère recrudescence de 1975 à 1976, avant de retrouver en 1977 la courbe descendante de 1975. Notons que le nombre

1. Hans-Heiner Kühne/Koichi Miyazawa, Kriminaliuät und Kriminalitätsbekämpfung in Japan, Versuch einer soziokulturell-kriminologischen Analyse, Sonderband der BKA-Forschungsreihe, Wiesbaden 1979. 
des délits connus a diminué de 1970 à 1974, malgré la croissance à la fois de l'ensemble de la population et de la population ayant atteint la majorité pénale. L'étude de l'évolution des cas pour les diverses infractions montre que l'augmentation des délits en valeur absolue depuis 1974 est essentiellement due à une importante progression du nombre des vols et des abus de confiance. Malgré cela, il convient de remarquer que la fréquence appliquée à la fois à l'ensemble de la population et aux citoyens ayant atteint la majorité pénale, et qui, seule permet de rendre compte de l'intensité de la criminalité dans un pays donné, a constamment diminué de 1970 à 1977 à l'exception du dérapage de 1975 a $1976^{2}$.

En 1978 cette tendance se confirme. Le nombre des délits de violence a continué de régresser au cours de cette année. Assassinat : $8,3 \%$; vol avec violence : $7,8 \%$; incendie volontaire : $7,0 \%$; viol : $1,6 \%$ (tous ces pourcentages sont indiqués aussi en valeurs absolues).

Par contre on observe en 1978 une importante recrudescence en matière de vol $(+5,9 \%)$ ainsi que de l'escroquerie $(+15,6 \%)$ et d'abus de confiance $(+13,6 \%)$. En raison de la fréquence de ces délits, le nombre des infractions en valeur absolue a progressé entre 1977 et 1978 de $5,4 \%$.

Le tableau II fait clairement apparaître l'importante diminution de la criminalité grave. En ce qui concerne les homicides, pour lesquels les tentatives sont généralement nombreuses sur le plan international, il aurait été intéressant de distinguer les crimes consommés et non consommés. Malheureusement le Japon ne retient cette distinction que depuis 1979.

Dans ce tableau nous présentons l'évolution de la criminalité grave, de 1969 à 1977, à travers certaines catégories de délits. En outre nous avons établi deux index à partir des éléments indiqués dans les sources japonaises, l'index $\mathrm{n}^{\circ} 1$, relatif à la fréquence absolue des délits, est constituée à partir des délits constatés en 1969, l'indice de base étant 100 . Nous avons choisi l'année 1969 comme année de référence parce que la dernière augmentation de l'ensemble des délits se situe entre 1969 et $\mathbf{1 9 7 0 \text { , }}$ avant le brutal revirement qui s'annonçait pour la plupart des crimes graves dès 1969.

2. Pour détails, voir tableau I. 
TABLEAU I

Délits (infractions au code penal) et donnés relatives a la population

\begin{tabular}{|c|c|c|c|c|c|}
\hline Annto & $\begin{array}{l}\text { Population } \\
(\times 1000)\end{array}$ & $\begin{array}{l}\text { Nombre des infractions } \\
\text { conzues par les } \\
\text { services de la police }\end{array}$ & $\begin{array}{c}\text { Fréquence générale } \\
\text { (pour } \\
100000 \text { habitants) }\end{array}$ & $\begin{array}{l}\text { Personnes ayant atteint } \\
\text { la majorité pénale } \\
(\times 1000)\end{array}$ & $\begin{array}{l}\text { Fréquence pour } 100000 \\
\text { personnes ayant atteint } \\
\text { la majorité pénale }\end{array}$ \\
\hline 1948 & 80003 & 1603265 & 2004 & 53413 & 3002 \\
\hline 1953 & 87033 & 1344482 & 1545 & 58858 & 2284 \\
\hline 1955 & 89276 & 1478202 & 1656 & 61443 & 2406 \\
\hline 1964 & 97186 & 1609741 & 1656 & 73825 & 2181 \\
\hline 1968 & 101408 & 1742479 & 1718 & 78677 & 2215 \\
\hline 1969 & 102648 & 1848740 & 1801 & 79740 & 2319 \\
\hline 1970 & 103720 & 1932401 & 1863 & 80500 & 2401 \\
\hline 1971 & 105014 & 1875383 & 1786 & 81364 & 2305 \\
\hline 1972 & 107332 & 1818072 & 1694 & 82947 & 2192 \\
\hline 1973 & 108710 & 1728726 & 1590 & 83885 & 2061 \\
\hline 1974 & 110049 & 1671947 & 1519 & 84792 & 1972 \\
\hline 1975 & 111934 & 1673727 & 1495 & 86323 & 1936 \\
\hline 1976 & 113086 & 1691229 & 1496 & 87195 & 1939 \\
\hline 1977 & 114154 & 1704995 & 1494 & 88145 & 1934 \\
\hline
\end{tabular}

3. La majorité pénale est acquise à partir de la $15^{\circ}$ année. Article 41 du code pénal japonais. 
TABLEAU II

Fréquence des délits de violence

\begin{tabular}{|c|c|c|c|c|c|c|c|c|c|c|c|c|c|c|c|}
\hline \multirow[t]{2}{*}{ Anné } & \multicolumn{3}{|c|}{$\begin{array}{l}\text { Homicides } \\
\text { volontaires }\end{array}$} & \multicolumn{3}{|c|}{ Vol avec violence } & \multicolumn{3}{|c|}{$\begin{array}{l}\text { Vol avec viol, coups } \\
\text { et blessures ou } \\
\text { suivi de mort }\end{array}$} & \multicolumn{3}{|c|}{ Chantage } & \multicolumn{3}{|c|}{$\begin{array}{l}\text { REunions armées } \\
\text { prohibées }\end{array}$} \\
\hline & Nombre & $\begin{array}{c}\text { Index } \\
1\end{array}$ & $\begin{array}{c}\text { Index } \\
2\end{array}$ & Nombre & $\begin{array}{c}\text { Index } \\
1\end{array}$ & $\begin{array}{c}\text { Index } \\
2\end{array}$ & Nombre & $\begin{array}{c}\text { Index } \\
1\end{array}$ & $\underset{2}{\text { Index }}$ & Nombre & $\begin{array}{c}\text { Index } \\
1\end{array}$ & $\underset{2}{\text { Index }}$ & Nombre & $\begin{array}{c}\text { Index } \\
1\end{array}$ & $\underset{2}{\text { Index }}$ \\
\hline 1969 & 2098 & 100 & 2,63 & 1414 & 100 & 1,77 & 1310 & 100 & 1,64 & 18204 & 100 & 28,8 & 325 & 100 & 0,41 \\
\hline 1970 & 1986 & 95 & 2,47 & 1451 & 103 & 1,80 & 1238 & 95 & 1,54 & 18775 & 103 & 23,3 & 219 & 67 & 0,27 \\
\hline 1971 & 1941 & 93 & 2,39 & 1275 & 90 & 1,57 & 1164 & 89 & 1,43 & 17406 & 95 & 21,4 & 259 & 80 & 0,32 \\
\hline 1972 & 2060 & 98 & 2,48 & 1306 & 92 & 1,57 & 1194 & 91 & 1,44 & 16053 & 88 & 19,3 & 177 & 54 & 0,21 \\
\hline 1973 & 2048 & 98 & 2,44 & 1056 & 75 & 1,26 & 944 & 72 & 1,12 & 14652 & 80 & 17,5 & 180 & 55 & 0,21 \\
\hline 1974 & 1912 & 91 & 2,25 & 1121 & 79 & 1,32 & 1019 & 78 & 1,20 & 13852 & 76 & 16,3 & 222 & 68 & 0,26 \\
\hline 1975 & 2098 & 100 & 2,43 & 1219 & 86 & 1,41 & 1081 & 83 & 1,25 & 14255 & 78 & 16,5 & 170 & 52 & 0,19 \\
\hline 1976 & 2111 & 101 & 2,42 & 1143 & 81 & 1,31 & 952 & 73 & 1,09 & 11497 & 63 & 13,2 & 159 & 49 & 0,18 \\
\hline 1977 & 2031 & 97 & 2,30 & 1173 & 83 & 1,33 & 922 & 70 & 1,08 & 10904 & 60 & 12,4 & 162 & 50 & 0,18 \\
\hline
\end{tabular}

4. Le code pénal japonais ne distingue pas entre «assassinat » et emeurtre >; l'article 199 du code pénal japonais vise l'homicide volontaire de façon générale.

5. Article 208 du code pénal japonais : « En cas de réunion d'au moins deux personnes en vue d'un homicide, coups et blessures ou attentat contre la propriété, quiconque des participants aura des armes dangereuses ou aura connaissance qu'elles existent ou sont disponibles sera puni de la réclusion criminelle de deux ans au plus ou d'une amende de 500 Yen au plus. * 
L'index $\mathrm{n}^{\circ} 2$ reproduit pour les différents délits, la fréquence appliquée aux crimes majeurs.

Les délits (chantage et réunions armées prohibées) se font rares entre 1969 et 1970. Il en résulte des index 1 et 2 que ceci se vérifie aussi bien pour la fréquence absolue que pour la fréquence appliquée à l'ensemble des crimes majeurs. Quant au délit de la réunion armée prohibée, il est reconnu dés qu'il y a réunion - il s'agit pratiquement toujours de manifestations peu importe le nombre de participants.

Au vu de ces données, on pourrait présumer que le tableau ne refiète pas la réalité, parce que les données indiquées dans les statistiques de la police sont incomplètes ou retouchés afin de présenter des résultats favorables.

De tels soupçons sont pourtant sans fondement car nous avons constaté "que la police japonaise procède très consciencieusement à l'établissement de ses statistiques.

Néanmoins un point obscur subsiste. Les particularités de la structure sociale japonaise, dont nous traitons ci-dessous, ont un double effet : elles empêchent toute forme de déviation et en même temps la masquent lorsqu'elle existe. Ce qui expliquerait que le « chiffre noir » de la criminalité est nettement plus élevé, comme le confirme notre enquête effectuée à Tokyo?

\section{STRUCRURE SOCIALE ET DEVIATION}

a) La priorité du groupe

Le groupe est le concept central permettant de comprendre la forme de vie japonaise. Le Japonais pense et vit en tant que membre du groupe. Son individualité se reflète à l'intérieur des relations du groupe. Le Japonais accède à une compréhension de soi grâce à l'introspection qui s'effectue en même temps par les yeux du groupe.

La valeur de l'existence individuelle est fonction de l'utilite que représente l'individu pour le groupe, dans lequel il vit dans une dépendance réciproque. Certes les Occidentaux attachent également de l'importance aux répercussions du comportement d'un

6. Kühne et Miyazawa, S. 17-21.

7. Kuhne et Miyazawa, S. $76 \mathrm{ff}$, insbes. S. 82-90. 
individu sur ses prochains, lorsqu'il s'agit d'évaluer sa personne. Cependant ces répercussions ne font pas l'objet de l'évaluation, mais servent en quelque sorte uniquement d'indicateurs, car l'évaluation ne porte que sur la personne, prise isolément.

Le psychiatre Takeo Doi marque cette différence à l'aide du terme « jibun \ qui recouvre à peu près l'idée suivante : « le moi, la personnalité $\$$. Le Japonais s'efforce autant que possible d'adapter son * moi » ( jibun ») aux exigences du groupe, même et surtout au détriment de sa personnalité, alors que pour les Occidentaux, l'idéal consiste à se réaliser en tant qu'individu, de façon à se détacher du groupe, soit pour devenir son chef, soit pour vivre isolément, en toute indépendance du groupe ${ }^{2}$. De même en cas de promotion sociale, le Japonais doit généralement ses succès au groupe qui le porte en avant et auquel il reste toujours attaché. Peu importe l'importance de sa promotion, il ne sera jamais un chef de groupe au sens occidental du mot. L'histoire japonaise ne connaît pas de chefs charismatiques ${ }^{10}$, mais uniquement des personnes qui ont été attachées au groupe de façon particulièrement intense et qui, pour cette raison se sont hissées au sommet de la hiérarchie, fortement structurée. Cette position ne leur confère guère, aujourd'hui comme autrefois, le droit ou le pouvoir de prendre des décisions individuelles. Au contraire, cette position exige un renforcement des devoirs au sein du groupe puisque le consensus des membres du groupe doit être garanti de manière implicite ou explicite pour toute décision importante. Aussi n'est-il pas surprenant que les personnalités dirigeantes du monde des affaires se signalent, moins par des connaissances techniques exceptionnelles que par la faculté de provoquer au sein du groupe, de façon harmonieuse le consensus des membres pour les décisions en suspens. Le fait que chaque groupe soit fortement hiérarchisé ne modifie en rien la nécessité d'une participation active de tous les membres en vue d'une parfaite harmonie au sein du groupe (cf. infra b).

8. Takeo Doi, The Anatomy of Dependence, Tokyo, New York, San Francisco, 1973, 3, 1976, p. 132 et suivantes.

9. Nakane, Japanese Society, Berkeley, 1972, p. 150, donne une définition caractéristique de l'ambition des Japonais : \& Le Japonais ne vise pas un emploi supérieur, mais aimerait être l'égal de ses supérieurs. \$

10. I. Ben-Dasan, The Japanese and the Jews, New York, Tokyo, 1972, p. 51. La langue japonaise ne comporte pas de mot pour le concept de chef ou de leadership, cf. Nakane, supra, p. 69. 
Nous pouvons donc affirmer que le Japonais existe non pas en tant qu'être individuel, mais comme partie dépendante d'un ensemble. Les répercussions d'une telle compréhension de soi sur un éventuel comportement déviationniste sont évidentes. D'une part l'absence de toute perception égocentrique ne favorise guère un comportement déviant. D'autre part le Japonais, conditionné par son appartenance au groupe, appartenance qui se manifeste non seulement dans ses relations sociales externes, mais qu'il ressent également en son for intérieur, reste parfaitement maître de son comportement ; l'omniprésence du groupe, qui est à la fois effective et psychique, et surtout le désir d'adopter le comportement attendu par le groupe, interdisent tout acte positif ou négatif qui ne serait pas conforme. La vie privée, réduite à l'individu n'existe pas. Même au niveau du langage, on retrouve cet état de fait. Le Japonais ne connaît que la distinction interne et externe (en relation avec un groupe) et ignore la différence que nous établissons entre public ou collectif et privé ${ }^{11}$. La liberté n'existe qu'au sein du groupe. Le Japonais ne souhaite pas, il ne lui viendrait jamais à l'esprit de se libérer du groupe. La remarque de Clifford est pertinente à cet égard : * Si la liberté est le prix de la criminalité, nous constatons que les Japonais ne sont pas disposés à payer ce prix ${ }^{12}$.

Normalement le Japonais appartient à trois groupes qui déterminent sa vie : le premier lui est attribué dès la naissance, le clan familial ; le second résulte de la socialisation, la famille professionnelle (il s'agit du groupe de personnes, sur le lieu de travail ou de formation, auxquelles il est lié par l'organisation de l'entreprise ou de l'institution) ; le troisième groupe est constitué par le voisinage (tonarigumi) dont les liens sont moins contraignants. Les conséquences de cette concentration en groupes quant au comportement déviationniste - défini par la valeur de référence de chaque groupe - sont multiples. Le comportement des membres du groupe, dans la mesure où il se trouve dans le collimateur du groupe, est fortement bloqué. La dépendance psychique et les contrôles pratiques sont trop importants. La question de savoir s'il est possible de réduire par là-même le comportement criminel, dont la définition figure non pas au

11. Cf. Doi, supra, p. 43. Le concept * oyake s qui sert au public pour désigner l'Etat, se rapporte aussi à un groupe à savoir le famille impériale, la grande famille qui réunit tous les clans.

12. Clifford, Crime Control in Japan, Toronto, London, 1976, p. 8. 
répertoire des valeurs du groupe mais dans la loi, est fonction de l'orientation du groupe. Lorsqu'il n'y a pas concordance des définitions, comme cela se produit dans les nombreuses bandes ${ }^{13}$, dont l'organisation, calquée sur les entreprises légales de la vie économique, est structurée en associations familiales de type patriarcal, l'appartenance au groupe peut, dans une certaine mesure, favoriser les agissements délictueux à l'intérieur du groupe. Notamment pour les délits de coups et blessures. Notons cependant que nous retrouvons même ici, dans les relations internes, l'influence du groupe favorisant un comportement non criminel, car ces organisations criminelles doivent respecter, dans leur propre intérêt, les droits essentiels de leurs membres, ces mêmes droits que la loi pénale cherche à protéger. Les infractions qui seraient commises malgré la conformité de la valeur du groupe au droit pénal, sont réglées, pour la plupart, à l'intérieur du groupe. Ceci correspond à la logique de la vie de groupe japonaise. En effet le fait pour un étranger au groupe de prendre connaissance de ces \& pannes > internes, rendrait l'acte d'autant plus grave. Même et surtout en présence de délits graves, le groupe s'efforce de trouver une solution interne, d'indemniser les victimes et d'obtenir leur silence. Les services de police et le parquet lui sont reconnaissants et ne cherchent pas à être informés. $\mathrm{Si}$, néanmoins pour des motifs de politique criminelle, les autorités essaient d'instruire l'affaire, elles se heurtent à la structure du groupe et rencontrent d'énormes difficultés pour recueillir des informations. Voilà ce qui expliquerait l'importance particulière du * chiffre noir \$.

Par contre l'appartenance au groupe a des effets de prevention criminelle dans les relations avec les étrangers au groupe. Pour ce qui est des organisations criminelles, il est évident que l'appartenance au groupe provoque un accroissement des infractions.

Quant aux groupes dont la valeur de référence est conforme à la loi, l'analyse est plus complexe.

En principe tout étranger (tanin), c'est-à-dire la personne extérieure au groupe dont on fait partie, est traité sans grande considération, ni courtoisie. Il n'existe aucun lien avec le \& tanin >

13. Vgl. dazu etwa Miyawaki, Crack-down operations against organized Racketeer Groups \$, International Criminal Police Review, Interpol General Office, juin-juillet 1979 , p. 34 . 
qui vit en quelque sorte dans un autre monde. Peu importe donc la manière dont on se conduit à l'égard de l'étranger ${ }^{14}$. Ceci explique l'attitude brutale des Japonais, aux yeux des Occidentaux, allant à l'encontre de la légendaire courtoisie japonaise. Cette indifférence se manifeste dans le métro ou dans la rue lorsque l'étranger est bousculé par des passants pressés, sans que l'< auteur > ne daigne jeter un regard sur sa \& victime », encore moins murmurer un mot d'excuse. De même, la conduite des touristes japonais ${ }^{15}$, qui n'est pas particulièrement impregnée de réserves et de courtoisie, reflète les différences entre un \& tanin > et un membre du groupe. Des relations humaines ne s'établissent qu'à partir du moment où l'on cesse d'être un - tanin $>$.

Cette indifférence frisant le mépris à l'égard d'étrangers, favorise en principe l'accomplissement de délits à leur endroit. La résistance intérieure est diminuée. Seule la contrainte de déshonorer son groupe fera hésiter le membre. La conscience et le sentiment de culpabilité du Japonais se développent non pas selon le schéma moral individuel, mais d'après la morale collective du groupe ${ }^{x 6}$. Tout ce qui aura des effets néfastes pour le groupe sera qualifié de mal. Il convient de craindre de tels effets, suite aux agissements à l'encontre du * tanin », lorsqu'ils dépassent le domaine des infractions mineures.

L'appartenance au groupe crée un autre facteur de criminalité, dans la mesure où il s'agit avant tout de maintenir le groupe et de lui procurer le maximum d'avantages. Les violations de la loi en faveur du groupe seront justifiées par la morale du groupe tant que l'avantage obtenu sera jugé plus important que l'atteinte à l'honneur qui pourrait résulter en cas de découverte du délit. Parmi ces genres de criminalité figurent les délits contre les biens, les infractions économiques et la violation des règles protectrices de l'environnement. La technique de neutralisation ${ }^{17}$, qualifiée par Matza et Sykes, de \& référence à une instance supérieure, est particulièrement intéressante ici. Surtout dans une société où l'individu n'existe pas en tant que tel, mais se considère et est considéré comme partie intégrante d'un groupe, la

14. Voir Doi, S. 36 ff.

15. Cette particularité est visé par le vieux proverbe japonais : 4 Lo voyageur se débarrasse de toute pudeur.

16. Doi, S. $36 \mathrm{ff}$.

17. Sykes, Matza, * Techniques of Neutralisation, A Theory of Delinquency ", American Sociological Review, 1957, 22, S. 664 ff. 
référence à l'intérêt supérieur du groupe lors de l'accomplissement d'un délit, constitue, sur le plan psychologique, un argument très puissant à la décharge de son auteur.

\section{b) Structure du groupe}

La société japonaise, organisée en groupes, se caractérise par une très forte hiérarchisation. Avec une précision alarmante, chacun se voit attribuer une place déterminée qui lui permet de se démarquer par rapport aux autres qui se trouvent au-dessus linquency 3, American Sociological Review, 1957, 22, S. $664 \mathrm{ff}$.

ou en dessous de lui. Dans un groupe, l'égalité n'existe pas et selon un système subtil, mettant l'accent sur les grandes différences, chaque personne se trouve dans un rapport de dépendance ${ }^{18}$. Les critères essentiels de la classification hiérarchique sont fournis par les principes du \& sempai-kohai > et du * oyabunkobun ». Les notions « sempai-kohai » signifient tout simplement l'aîné - le cadet et expriment le principe d'aînesse dont la portée est pratiquement illimitée. L'âge en tant que principe de classe est fonction de la durée d'appartenance au groupe. Dans la famille entre parents consanguins, le principe d'aînesse repose sur l'âge. Pour les autres, par exemple entre parents par alliance, il repose sur l'entrée dans le groupe, donc l'époque du mariage. Dans la famille professionnelle, le point de départ est aussi l'entrée dans le groupe.

Le principe du rendement que les Occidentaux opposent au principe d'aînesse n'a pas réussi à s'implanter au Japon malgré des vives discussions. Il est pratiquement inconcevable pour un Japonais d'avoir un supérieur direct ayant moins d'années de service. Le rendement ou plutôt la capacité de rendement est une condition que chacun remplit sans problème. Pour une meilleure compréhension, il faut savoir que l'accès à une profession est défini clairement par des qualifications formelles, auxquelles le candidat doit pleinement satisfaire et que le schéma de l'avancement est déterminé d'avance. Le risque que des personnes non qualifiées accèdent à des fonctions de responsabilité par l'ancienneté, est pratiquement écarté. En même temps, cela signi-

18. Dans un grand groupe, tel que l'entreprise, il existe naturellement des personnes occupant le même rang hiérarchique (doryo). Mais ces grands groupes sont divisés en sous-groupes qui ne connaissent pas de \& doryo *. Les relations horizontales des « doryo * dans les grands groupes sont pratiquement inexistantes. Seule la relation verticale importe. 
fie qu'à qualifications professionnelles égales, ce n'est pas le meilleur mais l'aîné qui bénéficie de l'avancement. D'après l'idéologie occidentale du rendement, un tel système est douteux et impropre aux durs combats concurrentiels auxquels sont soumis les employeurs - quoique en réalité, le principe du rendement comporte également un bon nombre d'exceptions sous la forme de protection, du principe d'aînesse, voire du principe d'incompétence. Pour revenir à la fonction particulière des dirigeants japonais des groupes de sociétés, il faut préciser que l'exclusion du principe de rendement lors de l'avancement n'a pas, ou peu, d'effets néfastes. Chaque emploi, jusqu'aux plus élevés, est marqué au Japon par un renforcement des obligations envers le groupe. Il est impossible pour un dirigeant de prendre des décisions individuelles. Une telle personne - comme d'ailleurs toutes les autres - reste dépendante de la décision du groupe ${ }^{19}$.

Certes, vis-à-vis des tiers, le chef du groupe détient le pouvoir de décision, mais dans les relations internes, il ne peut décider seul. Toute décision nécessite le consensus du groupe. A cet égard, deux choses sont importantes : en premier lieu le consensus doit être obtenu dans le respect de l'harmonie du groupe et donc, en second lieu la décision doit être adoptée à l'unanimité. Par conséquent il appartient au chef du groupe de veiller à l'occasion de chaque décision au maintien de cette harmonie. Peu importe finalement le sens de la décision, car la responsabilité incombe toujours au groupe tout entier, qui devra en supporter les conséquences.

Pour les Japonais, il est capital de manifester l'harmonie extérieure. Cet effort trouve son fondement dans la notion aningen > qui signifie à peu près humanité, ou leçon d'humanité. c Ningen > est le mot clef à l'aide duquel les Japonais parviennent à un consensus malgré toutes les divergences et même en dépassant le cadre du groupe. Pour Dasan, «ningen * est le principe qui gouverne le Japon et maintient les Japonais unis, mieux que toute loi $^{20}$.

Il importe de noter que malgré le souci d'harmonie et le souci d'obtenir un consensus, la hiérarchie rigide reste intacte. Au contraire c'est bien grâce à la structure verticale du groupe

19. Nakane, p. 150.

20. Ben-Dasan, p. 100. 
que le consensus peut être obtenu en toute harmonie, car la discussion précédant la décision sera menée en fonction du rang occupé. Même sans l'intervention expresse d'un * sempai > (aîné) un « kohai > ne prendra jamais la parole avant que ses supérieurs n'aient parle. De plus dans sa formulation, un \& kohai > n'ira jamais à l'encontre de l'opinion d'un < sempai *. (Ningen > est la garantie que la discussion ne dégénère pas en dispute, car l'impossibilité voire l'incongruité de contredire ouvertement un aîné, oblige ce dernier à prendre d'emblée en considération les intérêts des jeunes. S'il ne le fait pas, il fait preuve d'un manque de < ningen > et compromet l'harmonie du groupe. En effet les propos du «sempai " qui ne sont pas conformes à l'intérêt général de tous les membres du groupe, ne manqueront pas, malgré l'approbation formelle des * kohai », d'engendrer des frustrations. Et c'est justement ce qu'on cherche à éviter par le maintien de l'harmonie au sein du groupe.

Le principe d'aînesse est fortement renforcé par la relation d'< oyabun > d « kobun ». « Oyabun » signifie une personne avec « oya », c'est-à-dire le statut de parents. Kobun 》 signifie une personne avec \& ko «, c'est-à-dire le statut d'enfant. Par « oyabun \$ on vise également le fait qu'une personne soit le précepteur d'une autre. On place donc sur le même plan la dépendance consanguine et la dépendance éducative. L's oyabun $\gg$ est naturellement l'aîné (\& sempai »). Il fait l'objet d'un respect particulier de la part du « kobun s, qui est en même temps le cadet, le « kohai \$. Par-delà les relations normales du groupe, l'e oyabun * et le c kobun s sont étroitement liés entre eux dans des rapports rappelant ceux du maître et de ses disciples chez les anciens Grecs. Il est inconcevable pour un a kobun \$, alors même que sa compétence et sa notoriété dépasseraient celles de son maître, de contredire ce dernier, surtout en public. L'obéissance qui frise déja la soumission dans les simples rapports esempai-kohai », est totale à l'égard du * oyabun \$.

Cependant l'étude du concept de l'harmonie du groupe montre que l'obéissance ne représente qu'un aspect des obligations, incombant aux membres du groupe. Les services, effectués dans la discipline sont minutieusement enregistrés, en vue d'être rétribués à leur juste valeur. « Hoon > signifie tenir ses propres engagements s et symbolise un système dans lequel on veille attentivement à ce que chaque service se compense de manière 
Equitable. Peu importe à quel moment la compensation intervient. Un bienfait oblige même les enfants ou les petits-enfants du bénéficiaire qui n'aurait pas eu l'occasion de le rendre en retour ${ }^{21}$.

Dans ce monde marqué par des relations verticales, où chacun se voit attribuer sa place de façon purement formelle, il n'est pas surprenant que la discussion autour du \& Labelling approach > reste sans écho. Pour un Japonais, il est tout naturel qu'on le couvre d'une certaine étiquette pour le classer dans la société. L'autorité du groupe qui attribue l'étiquette ainsi que le bien-fondé de l'étiquette ne sont jamais mis en cause, parce que la personne concernée se considère comme partie intégrante du groupe. Les différences de classe n'existent pratiquement pas et là où elles existent, elles ne sont pas ressenties en tant que telles. Dans la mesure où les individus sont classés par groupes, les particularités existantes ne sont pas interprétées en vue d'une différenciation par classes. L'intégration au sein des groupes s'oppose à toute idée de classes sociales. De plus le système fortement hiérarchisé, ne s'accommode pas de la catégorie jugée trop peu nuancée de la classe sociale.

L'ensemble de la démarche critique du \& Labelling approach s reste incompréhensible au Japon, ou du moins sans grand intérêt. Malgré les très bonnes connaissances des criminologues japonais quant à l'état des travaux scientifiques en Europe et en Amérique, et malgré un esprit très ouvert aux idées scientifiques occidentales, l'analyse du c Labelling approach > est pratiquement inconnue au Japon.

L'étude du eningen s, principe consistant à tenir compte de tous les intérêts en présence dans un souci d'harmonie au sein du groupe, nous a permis de mettre l'accent sur une caractéristique essentielle, venant atténuer la rigueur du système hiérarchisé. Nous allons maintenant, à partir du concept (amae >, développer un autre principe capital, allant dans le sens d'un renforcement des relations du groupe. Il s'avère difficile de traduire le mot « amae \$. La traduction la plus fidèle serait * amour passif, certitude d'être aimé .

Selon le psychiatre Takeo Doi, dans son importante analyse du concept * amae $>$, cette notion trouverait son origine dans le rapport parents-enfants. Un enfant fait preuve d'< amae > lors-

21. Nakane, p. 28. 
qu'il peut compter en toute confiance sur l'affection et sur l'indulgence de ses parents ${ }^{22}$. La dépendance des enfants à l'égard des parents devient supportable grâce à « amae ». L'adulte, intégré dans la structure verticale de la société, retrouve ou plutôt prolonge son enfance dans la mesure où il respecte comme ses parents, les personnes qui occupent un rang supérieur dans la hiérarchie du groupe ; mais en retour il attend d'elles l'indulgence et une sage conduite. C'est ainsi que, grâce à « amae », l'individu, en dépit d'une dépendance strictement délimitée dans la hiérarchie du groupe à l'égard des supérieurs, est accepté par ces derniers avec beaucoup d'indulgence. (Amae \ donne au subalterne l'impression, pleinement justifiée, d'être aimé et compris en raison justement de ses faiblesses et de ses erreurs.

Dans la vie réglée d'un Japonais, une telle certitude a valeur de véritable thérapeutique psychologique. L'échec ne le déshonorera pas forcément, mais sera accepté sans rancune. Ce qui ne signifie pas que tout manquement soit admis. En effet, alors même que le manquement fautif ne serait pas sanctionné par les supérieurs, son auteur devra s'efforcer d'adopter un comportement conforme au groupe. Néanmoins il ne faudrait pas voir dans ce sentiment de responsabilité un besoin de compensation du groupe, ce qui équivaudrait à une sanction indirecte. Au contraire l'auteur du fait ressent, comme l'enfant qui ayant commis une faute n'aurait pas été puni par ses parents mais réconforté, de la gratitude ce qui motive les efforts que demande à l'avenir une conduite irréprochable. Cette sécurité conférée par \& amae > n'est pas en contradiction avec le nombre de suicides dus au fait que la personne n'a pas supporté l'échec. Il n'arrive pratiquement jamais que le groupe refuse d'accorder * amae ». Même pour les actes criminels graves, le responsable trouve le soutien de son groupe à la seule condition de présenter des excuses. Si le responsable se suicide malgré tout, c'est parce qu'il est guidé par les mêmes sentiments que l'auteur de délits de moindre gravité qui s'efforce d'avoir une conduite irréprochable : à savoir la gratitude et le sentiment de responsabilité à l'encontre du groupe. Le responsable est persuadé que son délit est trop grave pour le

22. Doi, supra, p. 75, définit \& amae s par la tentative psychologique de nier la séparation de l'enfant et de la mere. Pour cette raison, la connaissance de l'autre personne et par là-même de sa propre personnalitó passe par une victoire sur * amae $\rangle$. Or comme le principe du amae domine au Japon, il y aurait là une explication. 
groupe et qu'il ne mérite pas l'\& amae ». Par son suicide il peut sauver ou rétablir à titre posthume l'honneur du groupe et de sa propre personne.

Le lecteur occidental, familiarisé avec la philosophie chrétienne de l'amour du prochain, ne manquera pas d'être surpris par le concept «amae », car il retrouve des principes connus mais issus d'une toute autre philosophie.

Les fondements de la tolérance résident dans les valeurs morales de la société japonaise. L'agressivité et l'idée de vengeance sont à bannir de la vie de société. A la différence des sociétés occidentales de rendement, od la condamnation de l'agressivité est battue en brèche chaque fois que cette dernière conduit au succès, le Japon dénie tout comportement agressif. Les relations humaines au Japon, même dans la vie des affaires, sont beaucoup moins tendues que dans les pays occidentaux ${ }^{23}$.

Ce manque d'agressivité, ainsi que l'absence d'une prise de position affective à l'encontre du délinquant, expliquent qu'au Japon toutes les théories criminelles, reposant sur le principe de la projection de la faute, demeurent sans objet. Les travaux de Freud sur la criminologie psychanalytique qui ont pour fonde. ment la théorie du bouc émissaire, n'ont connu aucune résonance au Japon. Dans la mesure où l'auteur de l'infraction ne suscite aucune haine, on ne peut pas, au sens de la théorie psychanalytique, projeter sur lui le surplus des agressions de la société. - Amae > représente pour les Japonais une soupape permanente et institutionalisée, leur permettant de se libérer des agressions, de sorte qu'ils ne connaissent pas de frustrations provoquées par l'interdiction d'aller à l'encontre de la loi. Les éléments de base sur lesquels repose la théorie du bouc émissaire font défaut. Voilà pourquoi les criminologues japonais n'ont pas retenu cette theorie.

III. LES SERVICES DE POLICE EN TANT QU'INSTANCB DE PREVENTION ET DE RÉPRESSION DE LA CRIMINALITÉ

Le taux des affaires élucidées par la police japonaise est très élevé : pour la période entre 1969 et 1978 et pour les délits

23. Voir : S. Hirakawa, A Case of Sympathetic Understanding of the Inner Life of Japan \$, The Japanese News Letters, Band VI, Nr. 5, 1979, S. 15. 
Taux des affaires élucidés en pourcentage

(Les chiffes entre parenthèses correspondent au taux des affaires élucidées en Allemagne)

\begin{tabular}{llllllllllllll}
\hline \multicolumn{1}{c}{ D\&lits } & 1969 & 1970 & 1971 & 1972 & 1973 & 1974 & 1975 & 1976 & \multicolumn{1}{c}{1977} & & 1978 \\
\hline Assassinat & 96,9 & 97,0 & 95,4 & 97,5 & 96,0 & 96,1 & 96,5 & 96,4 & 96,9 & $(95,8)$ & 96,9 & $(96,4)$ \\
Vol avec violence & 83,6 & 81,0 & 80,6 & 82,0 & 80,6 & 79,9 & 79,7 & 80,6 & 81,1 & $(52,3)$ & $\mathbf{7 8 , 0}$ & $(54,3)$ \\
Incendie volontaire & 80,1 & 85,4 & 81,9 & 87,6 & 83,8 & 86,4 & 86,4 & 88,2 & 84,0 & $(53,5)$ & 87,8 & $(53,3)$ \\
Viol & 92,7 & 92,0 & 91,6 & 92,7 & 91,2 & 91,3 & 91,5 & 91,7 & 90,0 & $(72,7)$ & 90,0 & $(72,4)$ \\
Acte de violence & 92,1 & 92,1 & 91,7 & 92,7 & 92,6 & 92,8 & 93,4 & 93,8 & 93,7 & $(-)$ & 93,7 & $(-)$ \\
Menace & 94,6 & 94,3 & 94,6 & 94,6 & 94,6 & 92,0 & 90,5 & 93,3 & 94,2 & $(-)$ & 95,1 & $(-)$ \\
Coup et blessure & 91,7 & 90,8 & 91,3 & 91,7 & 92,0 & 92,5 & 93,0 & 93,3 & 93,6 & $(88,7)$ & 94,6 & $(88,1)$ \\
Chantage & 80,4 & 83,2 & 83,0 & 83,9 & 85,4 & 83,9 & 85,1 & 86,3 & 87,2 & $(57,2)$ & 86,4 & $(58,5)$ \\
Vol simple & 45,0 & 47,5 & 48,2 & 50,1 & 50,5 & 51,1 & 51,6 & 53,8 & 51,1 & $(30,1)$ & 52,7 & $(30,1)$ \\
Escroquerie & 89,7 & 90,4 & 89,6 & 91,0 & 92,1 & 93,1 & 93,0 & 93,4 & 92,9 & $(95,7)$ & 95,2 & $(95,8)$ \\
Abus de confiance & 93,7 & 95,0 & 95,0 & 95,7 & 96,7 & 97,3 & 97,4 & 98,4 & 98,3 & $(82,9)$ & 98,7 & $(81,5)$ \\
Abus de blanc-scing & 99,7 & 99,7 & 99,0 & 98,1 & 96,5 & 99,1 & 99,6 & 96,1 & $101,3 *$ & $(99,7)$ & 96,8 & $(98,5)$ \\
& & & & & & & & & & & &
\end{tabular}

- Le taux des affaires flucidées peut dépasser 100\% car il englobe des affaires de l'an passé mais f́lucidfes l'annte de reférence. 
graves (assassinat, vol avec violence, incendie volontaire, viol, coups et blessures) la moyenne est de $86 \%$.

L'amélioration de l'efficacité du travail de la police a de multiples raisons : par exemple le niveau élevé de formation, l'importance du prestige social, une régression de l'agressivité. Notre étude se limitera à un seul principe d'organisation assurant la prévention et la répression : à savoir le c koban $*$ s.

Le Japon comporte une multitude de petits postes de police, répartis sur l'ensemble du territoire, quadrillé en petites zones facilement contrôlables 24 heures sur 24. Il existe environ 17000 de ces « koban \$. Le « koban 》 repose sur cette idée, également réintroduite en Allemagne, edu policier au coin de chaque rue », en rapport étroit avec les citoyens. Les a koban > sont placés en plein centre d'activités de leur zone de contröle. Dans les grandes villes, on les trouve généralement près des stations des transports en commun. Ceci facilite les contacts avec les gens qui se rendent à leur travail et qui y passant au moins deux fois par jour comme avec les autres habitants, tels les ménagères et les enfants, car autour de ce point central se développent à la fois les centres commerciaux et de loisirs. Dans les « koban », les agents sont de faction 24 heures sur 24. Is travaillent en trois équipes, effectuant 56 heures par semaine. La tâche essentielle pour un agent du « koban » est d'apprendre à connaître les habitants de la circonscription. A cette fin, les policiers rendent visite au moins deux fois par an aux ménages et aux petites entreprises. A l'occasion de ces visites, on aborde non seulement des problèmes généraux telle la protection de la maison contre les vols ou les manceuvres des escrocs, mais aussi des questions plus personnelles, a savoir les habitudes de vie des membres de la famille, leurs problemes et leurs rapports avec le voisinage. Les citoyens sont invités à exprimer leurs soupçons et leur méfiance à l'égard d'irregularités constatées dans leur entourage. Une telle attitude n'est pas qualifiée de dénonciation, mais traduit le souci de contribuer à l'intérêt général. L'agent est considéré comme l'autorité compétente pour le maintien de l'ordre et de l'harmonie au sein du groupe du voisinage (tonari gumi).

24. D'après D.H. Bayley, Force of Order. Pollce Behaviour in Japan and the United States, Berkeley and Los Angeles, 1976, Paperback 1978, p. 13-32. 
Dans l'hypothèse où à l'occasion de ses visites répétées l'agent ne rencontre personne au domicile, ce qui est relativement rare, il laissera dans la boîte à lettres un formulaire à remplir et à déposer au « koban ». En général on y donne suite.

Notre enquête ${ }^{25}$ a également confirmé que la grande majorité des Japonais est favorable à ces enquêtes intensives et régulières effectuées par la police et y répond volontiers. Ceci est probablement da au fait que, en ce qui a trait aux problèmes relatifs à leur vie privee, les Japonais peuvent compter sur l'assistance et les conseils du < koban », qu'il s'agisse de conflits conjugaux ou juridiques liés à la conclusion d'un contrat.

Les avantages d'un tel système de visite sont évidents. C'est ainsi, par exemple, qu'une personne, faisant l'objet de recherches, ne peut se terrer pendant plus d'une journée. En effet, pour les Japonais, rechercher plusieurs personnes cachées à l'intérieur du pays, est un problème secondaire, qui ne demande pas plus d'une semaine dans les cas les plus complexes.

Les informations recueillies par les agents du e koban s, à l'occasion de leurs visites présentent encore un autre intérêt.

Les villes japonaises déconcertent quelque peu le visiteur occidental de par leur aménagement sous forme de labyrinthes, dépourvu de toute organisation. Comme il n'existe pas en principe de noms de rues, comme la répartition se fait par circonscription ou par pâtés de maisons, comme la numérotation de ces unités ne s'établit pas selon leur alignement dans la rue mais en fonction de l'époque de leur construction, la recherche d'une adresse devient même pour un Japonais une véritable aventure. Pour cette raison, le Japonais joint toujours à son adresse un petit schéma indiquant le chemin au visiteur. Ni le conducteur de taxi, ni les pompiers, ni les policiers dépendant d'un autre « koban », ne pourront tout de suite trouver la maison, à partir de la simple indication de l'adresse. La complexité du système d'adresses japonais est telle qu'un nouvel agent du k koban * a besoin d'environ deux années pour connaître parfaitement son district, bien qu'il ne recouvre pas plus de $20 \mathrm{~km}^{2}$ dans les grandes villes. L'agent du * koban », en service extérieur, ne se contente pas de

25. Kühne, Miyazawa, supra, p. 142. Selon notre enquête, $77 \%$ des habitants interrogés étaient favorables à la visite à domicile des policiers pour qu'ils les questionnent sur leur âge, leur travail et leur famille. 
rendre visite aux citoyens de son district ou d'indiquer le chemin. Il doit, de jour comme de nuit, effectuer des rondes, dont la durée ne saurait être inférieure à six heures. Qu'il se déplace à pied ou à vélo, sa présence est ressentie dans toutes les parties du district à tout moment de la journée et de la nuit.

L'idée d'une omniprésence et d'une aimable serviabilité est très efficace et atteint son objectif de prévention et de répression de la criminalité.

\section{BIBLIOGRAPHIE}

BAYLEY, D.H. (1970) : Forces of Order. Police Behavior in Japan and the United States, Berkeley.

BEN-DASAN I. (1972) : The Japanese and the Jews, New York, Tokyo.

BENDIX, R. : « Preconditions of Development : A Comparison of Japan and Germany \, Dore, 1967.

CLIFFORD, W. (1976) : Crime Control in Japan, Toronto, London.

DANDO, Sh. : :Strafrechtsentwicklung in Japan nach dem Kriege?, ZStW 66 (1954). 151 ders.

-, * System of Discretionary Prosecution in Japan $»$, American Journal of Comparative Law, vol. 18, no 3, p. 521.

Dol, Takeo (1976) : The Anatomy of Dependence, Tokyo, New York, San Francisco, 1973, 3.

DORE, R.P. (1967) : Aspects of Social Change in Modern Japan.

HEARN, L. (1904) : Japan, Grosset and Dunlap - MacMillan, New York.

HIRAKAWA, S. (1979) : « A Case of Sympathetic Understanding of the Inner Life in Japan ", The Japan News Letter, vol. VI, no 5, p. 15.

KUHNE, Hans-Heiner ; MIYAZAWA, Koichi (1979) : Kriminalität und Kriminalitätbekämpfung in Japan. Versuch einer soziokulturellkriminologischen Analyse, Sonderband der BKA-Forschungsreihe, Weisbaden. 
LYMAN, St. (1961) : The Structure of Chinese Soctety in 19th Century America, Ph.D. Thesis, University of California.

MEHREN, A.T. (1969) : Law in Japan. The Legal Order in a Changing Society, Cambridge (Mass.), Tokyo.

MIYAWAKI, R. : \& Crack-down Operations against Organized Racketeer Groups *, International Criminal Police Review, Interpol General Secretariat, June/July 1979 , no 329.

NAKAMURA, H. (1979) : The Acceptance of Man's Natural Disposition 》, Japan Foundation Newsletters June-July 1979, p. 1-8.

NAKANE, Ch. (1972) : Japanese Society, Berkeley.

NATIONAL STATEMENT BY JAPAN : * Crime Prevention and Control - The Challenge of the Last Quarter of the Century 》.

SUZUKI, D. (1971) : Zen and Japanese Culture, Princeton.

TAKAYANAGI, K. and BLAKEMORE, Th. L. : A Century of Innovation : the Development of Japanese Law 1868-1961.

TREGER, H. (1975) : The Police/Social-Worker-Team. A New Model for Interprofessional Cooperation : a University Demonstration in Man-Power Training and Development, Springfield (IIl.). 